\title{
Histological subtype of lung cancer affects acceptance of illness, severity of pain, and quality of life
}

This article was published in the following Dove Press journal: Journal of Pain Research

\author{
Jacek Polański' \\ Mariusz Chabowski ${ }^{2,3}$ \\ Beata Jankowska-Polańska ${ }^{4}$ \\ Dariusz Janczak ${ }^{2,3}$ \\ Joanna Rosińczuk ${ }^{5,6}$ \\ 'Lower Silesian Oncology Center, \\ Home Hospice, Wroclaw, Poland; \\ ${ }^{2}$ Division of Surgical Procedures, \\ Department of Clinical Nursing, \\ Faculty of Health Science, Wroclaw \\ Medical University, Wroclaw, Poland; \\ ${ }^{3}$ Department of Surgery, 4th Military \\ Teaching Hospital, Wroclaw, Poland; \\ ${ }^{4}$ Department of Clinical Nursing, \\ Faculty of Health Science, Wroclaw \\ Medical University, Wroclaw, Poland; \\ ${ }^{5}$ Department of Nervous System \\ Diseases, Faculty of Health Science, \\ Wroclaw Medical University, Wroclaw, \\ Poland; ${ }^{6}$ Department of Clinical \\ Nursing, Faculty of Health Science, \\ Wroclaw Medical University, Wroclaw, \\ Poland
}

Correspondence: Mariusz Chabowski Department of Surgery, 4th Military Teaching Hospital, 5 Weigla Street, 50-98I Wroclaw, Poland

Tel +4826I 660247

Fax +48 261 660245

Email mariusz.chabowski@gmail.com
Introduction: Histologic classification of lung cancer plays an important role in clinical practice. Two main histological subtype of lung cancer: small-cell lung cancer (SCLC) and nonsmall-cell lung cancer (NSCLC) differ in terms of invasiveness, response to treatment, and risk factors, among others.

Aims: To evaluate differences in acceptance of illness, level of perceived pain, and quality of life (QoL) between patients with SCLC and NSCLC.

Materials and methods: Two hundred and fifty-seven lung cancer patients, who were treated in 2015, completed Acceptance of Illness Scale, Visual Analog Scale for pain, and European Organization for Research and Treatment of Cancer 30-item Core Quality of Life Questionnaire and European Organization for Research and Treatment of Cancer 13-item Lung Cancer specific Quality of Life Questionnaire. Clinical and sociodemographic data were collected. For statistical analysis, the Student $t$-test and the Mann-Whitney $U$ test were used. For comparisons among three or more groups, analysis of variance was employed.

Results: Patients with SCLC had significantly worse health as measured with the presence of metastases, parameters of lung function, comorbidities, and number of previous hospitalizations. The Acceptance of Illness Scale score and Visual Analog Scale score were significantly worse in patients with SCLC than in those with NSCLC ( $24.58 \pm 8.73$ vs $27.05 \pm 9.06 ; p=0.046$ and $4.81 \pm 2.01$ vs $4.17 \pm 1.97$; $p=0.003$ ). Patients with SCLC achieved worse scores of all aspects of QoL than patients with NSCLC. Comparison with the reference values showed that all dimensions of functioning are impaired in patients with lung cancer regardless of its type; only the role functioning in patients with NSCLC remains unaffected.

Conclusion: Monitoring of QoL, personalized approach to treatment, and interventions for symptom management should be conducted in a tailored manner. Socioeconomic status in lung cancer patients, especially those suffering from SCLC, needs to be addressed.

Keywords: Acceptance of Illness Scale, Visual Analog Scale for pain, lung cancer, quality of life, QLQ-C30 and QLQ-LC13

\section{Introduction}

Histologic classification of lung cancer plays an important role in clinical practice. Determining histologic subtype of lung cancer helps to personalize treatment strategies and determine prognosis more precisely. Since 1967, when the first edition of histological classification was published by the World Health Organization, efforts have been made to improve the knowledge about associations between cancer histology and response to treatment options, underlying genetic disorders, risk factors, and survival, among others. ${ }^{1,2}$ 
The histological characteristic of cancer is based on architectural or cytological features of cancer cells and includes mitotic count, cell differentiation, and the presence of necrosis. There are two main histological subtypes of lung cancer: small-cell lung cancer (SCLC), which has an incidence of $15 \%-17 \%$ of all diagnosed lung cancers, and nonsmall-cell lung cancer (NSCLC), which has an incidence exceeding $85 \% .^{3,4}$ Despite their origin in the lung tissue, many differences exist between these two subtypes such as invasiveness, response to treatment options, and risk factors.

SCLC is diagnosed about nine times less often than NSCLC, but this subtype of cancer is responsible for high mortality due to its invasiveness and ease of metastasis. The median survival of patients with this cancer is about 6 months and the 1-year relative survival rate about 33\%. Low survival rates are a consequence of $70 \%$ of SCLC being diagnosed at extensive disease stage with hematogenous metastases, which limits the use of radical surgery. However, sensitivity to initial chemotherapy and radiotherapy does not improve outcomes due to frequent recurrence. ${ }^{6}$

The natural history of the disease is different for NSCLC. First, about one-third of patients present with limited-stage disease at the time of diagnosis. However, the median survival of patients with NSCLC based on National Lung Cancer Audit is 6.3 months, and prognosis is better for patients with good performance status and early stage of the disease. ${ }^{7}$ There are differences in outcomes between males and females, partly due to exposure to tobacco smoke. According to Visbal et al, ${ }^{8}$ estimated crude survival in men is $51 \%$ and $15 \%$ at 1 and 5 years, whereas in women, it is $60 \%$ and $19 \%$, respectively.

The differences in the natural course of the disease between SCLC and NSCLC may translate into severity of perceived symptoms and adaptation to the disease; therefore, the aim of the study was to evaluate differences in acceptance of illness, the level of perceived pain, and quality of life (QoL) between patients with SCLC and NSCLC.

\section{Materials and methods}

For the study, 257 lung cancer patients (115 women and 142 men) treated in the Lower Silesian Center of Lung Diseases in Wroclaw in 2015 were enrolled. Two hundred and eighty patients at admission to hospital were asked to complete Acceptance of Illness Scale (AIS), Visual Analog Scale for pain (VAS), and European Organization for Research and Treatment of Cancer 30-item Core Quality of Life Questionnaire (QLQ-C30) and European Organization for Research and Treatment of Cancer 13-item Lung Cancer specific Quality of Life Questionnaire (QLQ-LC13) questionnaires for the assessment of QoL. Twenty-three patients were excluded due to incompletely or incorrectly filled in questionnaires. Clinical data were collected from medical records. Sociodemographic data were sourced from the survey. The study was conducted after obtaining approval from the local Bioethics Committee at Wroclaw Medical University (No 507/2015). Each enrolled patient gave his/her written informed consent prior to entering the study.

In the study, the AIS questionnaire developed by Felton et $\mathrm{al}^{10}$ and adapted to Polish conditions was used. ${ }^{9}$ This scale can be used to evaluate acceptance of any illness among adult patients. The scale consists of 8 items - answers are measured with a 5-point Likert scale. The total score is from 8 to 40. A total score below 20 indicates poor acceptance of illness translating into psychological discomfort. Scores between 20 and 30 indicate a moderate level of acceptance, whereas above 30 indicates high acceptance of the disease. The scale showed high reliability in Polish population (Cronbach's $\alpha=0.85$; test - retest reliability $=0.64) .{ }^{9}$

The intensity of pain was measured with the VAS for pain. It is one of the most common tools for assessment of pain, because it is simple and intuitive. It includes only 1 item in the form of a $10 \mathrm{~cm}$ horizontal line with the two endpoints labeled: "no pain" and "maximum pain" on which patients mark a point corresponding to the severity of pain they feel. The result ranges from 0 to 10 , with higher score indicating greater intensity of pain. ${ }^{11,12}$

For the measurement of QoL, two questionnaires were used: the European Organization for Research and Treatment of Cancer QLQ-C30 and a supplementary module for lung cancer patients. ${ }^{13-15}$ The QLQ-C30 is a 30-item questionnaire that includes questions about global health status, functioning (physical, role, cognition, emotional, and social), and symptoms (fatigue, nausea/vomiting, pain, dyspnea, insomnia, appetite loss, constipation, diarrhea, and financial problems). In the first module of the scale, higher scores indicate better QoL, whereas in the second, higher scores indicate greater symptom burden, thus worse QoL. The QLQ-LC13 module is used to evaluate lung cancerspecific symptoms such as dyspnea; coughing; hemoptysis; sore mouth and tongue; dysphagia; peripheral neuropathy; hair loss; pain in the chest, arm, or other sites; and using analgesics. Items in both QoL scales are scaled to range from 0 to 100. The results of QLQ-C30 were compared with reference values calculated separately for SCLC and NSCLC. ${ }^{16}$ The QLQ-C30 was validated among the Polish cancer population. The interclass correlations ranged from 0.82 to 0.91 and Cronbach's $\alpha$ was $0.62 .{ }^{17}$ 
Data were statistically analyzed. Descriptive statistics were calculated. The Shapiro-Wilk test was used to analyze the distribution of the data. For comparisons between two groups with normal distribution, Student $t$-test was used, whereas for comparisons between two groups with distribution other than normal, the Mann-Whitney $U$ test was used. For comparisons of quantities variables among three or more groups, analysis of variance was performed, that is, the Kruskal-Wallis test for variables with distribution other than normal. If such a comparison revealed significant differences, analysis of variance was followed by post hoc test: LSD Fisher test for variables with normal distribution and Dunn test for variables with distribution other than normal. Correlations were described as Pearson's correlation coefficient and Spearman's rank correlation coefficient depending on the distribution of variables. Data were considered to be statistically significant at a value of $p<0.05$. Statistical analysis was carried out with the R Project for Statistical Computing v. 3.4.1.

\section{Results}

Two hundred and fifty-seven lung cancer patients with mean age of 63.2 (9.4) were recruited for the study. Of this group, 72 were diagnosed with SCLC and 185 with NSCLC. No significant differences were found between the group with SCLC and NSCLC in age (62.8 \pm 9.6 vs $64.1 \pm 8.8 ; p=0.358)$, $\operatorname{sex}(p=0.841)$, marital status $(p=0.701)$, education $(p=0.054)$, employment status $(p=0.279)$, performance status $(p=0.325)$, and the number of reported symptoms $(p=0.353)$. Patients with SCLC had significantly more comorbidities $(p<0.001)$, more metastases $(p=0.036)$, and were more often admitted to the hospital $(p<0.001)$. Significantly greater number of patients with NSCLC than with SCLC was treated with surgery as the only type of treatment ( $40.54 \%$ vs $6.94 \%$; $p<0.001)$. Over $93 \%$ of patients with SCLC were offered other types of treatment including combined treatment. Additionally, patients with SCLC had lower values of forced expiratory volume in 1 second $(2.1 \pm 0.6$ vs $2.4 \pm 0.8 ; p=0.014)$ and forced vital capacity ( $2.8 \pm 0.9$ vs $3.1 \pm 1 ; p=0.005)$, but the difference in forced expiratory volume in 1 second/forced vital capacity (\%) was insignificant $(79.2 \pm 15.879 .4 \pm 20.6$; $p=0.219$ ). Demographic and clinical characteristics by the type of the cancer histology are presented in Table 1.

AIS score was significantly lower in patients with SCLC than in those with NSCLC $(24.58 \pm 8.73$ vs $27.05 \pm 9.06$; $p=0.046$ ). This result showed worse acceptance of illness in the group of SCLC patients. Similarly, patients with SCLC experienced significantly more pain measured with VAS than patients with NSCLC $(4.81 \pm 2.01$ vs $4.17 \pm 1.97 ; p=0.003)$.
For both groups, AIS score was significantly negatively correlated with VAS score $(R=-0.517 ; p<0.001)$, which means that less pain is associated with better acceptance of illness.

Patients with SCLC had lower QoL than patients with NSCLC. Nearly all of the aspects of QoL were affected by the disease to the greater degree in patients with SCLC than in those with NSCLC. Comparison with the reference values showed that all dimensions of functioning are impaired in patients with lung cancer regardless of its type; only the role functioning in patients with NSCLC remains unaffected. Scores of QLQ-C30 with respective reference values and scores of QLQ-LC13 scale are presented in Table 2.

The level of acceptance of illness correlates with QoL. There was a significant correlation between AIS score and all functioning scores measured with QLQ-C30 in both study groups. Severity of greater number of symptoms was significantly correlated with AIS score in the group of patients with NSCLC than in the group of patients with SCLC. Correlation coefficients are presented in Table 3.

\section{Discussion}

Our study revealed that patients with SCLC had significantly worse health condition, lower acceptance of illness, and experienced significantly more pain, which was associated with worse QoL in comparison with patients with NSCLC; however, comparison with reference values revealed that lung cancer patients reported reduced QoL regardless of the cancer subtype in all QLQ-C30 items except for role functioning in NSCLC patients. ${ }^{16}$

Patient-reported QoL is an important aspect of treatment in oncology. The Food and Drug Administration included QoL into patient-reported outcomes and defined them as a measurement of any aspect of a patient's health status that comes directly from the patient (ie, without the interpretation of the patient's responses by a physician or anyone else). ${ }^{18}$ First of all, QoL has an impact on prognosis. Montazeri et al ${ }^{19}$ confirmed that general health measured with the Nottingham Health Profile and functioning scores and global QoL assessed with QLQ-C30 are significant predictors of survival in the population of lung cancer patients. Efficace et al, ${ }^{20}$ using the same tools as we used in our study (QLQ-C30 and QLQLC13), showed that among patients with NSCLC stage IIIB and stage IV, pain and dysphagia translated into significant increase in the likelihood of death with an HR of $1.11(95 \% \mathrm{CI}$ $1.07-1.16 ; p<0.001)$ and 1.12 (95\% CI: $1.04-1.21 ; p=0.003$ ), respectively. Similar results were reported by Herndon et al ${ }^{21}$ who studied a group of patients with advanced metastatic NSCLC. After adjustment for clinical factors, perceived pain 
Table I Demographic and clinical characteristics of the study group

\begin{tabular}{|c|c|c|c|c|}
\hline Variables & SCLC; $n=72, n(\%)$ & NSCLC; $n=185, n(\%)$ & Total; $n=257, n(\%)$ & $p$-value \\
\hline \multicolumn{5}{|l|}{ Sex } \\
\hline Woman & 31 (43.06\%) & $84(45.41 \%)$ & II 5 (44.75\%) & \multirow{2}{*}{0.841} \\
\hline Men & $4 \mathrm{I}(56.94 \%)$ & I0I (54.59\%) & 142 (55.25\%) & \\
\hline \multicolumn{5}{|l|}{ Marital status } \\
\hline In a relationship & $45(62.50 \%)$ & 109 (58.92\%) & 154 (59.92\%) & \multirow{2}{*}{0.701} \\
\hline Alone & $27(37.50 \%)$ & $76(41.08 \%)$ & $103(40.08 \%)$ & \\
\hline \multicolumn{5}{|l|}{ Education } \\
\hline Primary & 14 (19.44\%) & 15 (8.1 I\%) & 29 (II.28\%) & \multirow{4}{*}{0.054} \\
\hline Vocational & $28(38.89 \%)$ & $90(48.65 \%)$ & 118 (45.91\%) & \\
\hline Secondary & $25(34.72 \%)$ & 61 (32.97\%) & $86(33.46 \%)$ & \\
\hline Higher & $5(6.94 \%)$ & $19(10.27 \%)$ & $24(9.34 \%)$ & \\
\hline \multicolumn{5}{|l|}{ Employment status } \\
\hline Employed & $14(19.44 \%)$ & $54(29.19 \%)$ & $68(26.46 \%)$ & \multirow{3}{*}{0.279} \\
\hline Pensioner & $56(77.78 \%)$ & 125 (67.57\%) & $|8|(70.43 \%)$ & \\
\hline Supported by relatives & $2(2.78 \%)$ & $6(3.24 \%)$ & $8(3.11 \%)$ & \\
\hline \multicolumn{5}{|l|}{ Comorbidities } \\
\hline No comorbidities & $12(16.67 \%)$ & $62(33.51 \%)$ & $74(28.79 \%)$ & \multirow{4}{*}{$<0.001$} \\
\hline One disease & $28(38.89 \%)$ & $82(44.32 \%)$ & $110(42.80 \%)$ & \\
\hline Two diseases & $25(34.72 \%)$ & 39 (21.08\%) & $64(24.90 \%)$ & \\
\hline Three diseases & 7 (9.72\%) & $2(1.08 \%)$ & $9(3.50 \%)$ & \\
\hline \multicolumn{5}{|l|}{ Metastases } \\
\hline No metastases & $35(48.61 \%)$ & $|2|(65.4 \mid \%)$ & 156 (60.70\%) & \multirow{3}{*}{0.036} \\
\hline To one organ & $23(31.94 \%)$ & $44(23.78 \%)$ & $67(26.07 \%)$ & \\
\hline Multiorgan & $14(19.44 \%)$ & $20(10.81 \%)$ & $34(13.23 \%)$ & \\
\hline \multicolumn{5}{|l|}{ Treatment } \\
\hline Surgery alone & 5 (6.94\%) & 75 (40.54\%) & $80(31.13 \%)$ & \multirow{2}{*}{$<0.001$} \\
\hline Other & 67 (93.06\%) & $110(59.46 \%)$ & 177 (68.87\%) & \\
\hline \multicolumn{5}{|l|}{ WHO performance status } \\
\hline Grade 0 & $13(18.06 \%)$ & $34(18.38 \%)$ & 47 (I8.29\%) & \multirow{5}{*}{0.325} \\
\hline Grade I & 27 (37.50\%) & $80(43.24 \%)$ & $107(41.63 \%)$ & \\
\hline Grade 2 & $26(36.11 \%)$ & 61 (32.97\%) & $87(33.85 \%)$ & \\
\hline Grade 3 & $3(4.17 \%)$ & $9(4.86 \%)$ & $12(4.67 \%)$ & \\
\hline Grade 4 & $3(4.17 \%)$ & I (0.54\%) & $4(1.56 \%)$ & \\
\hline
\end{tabular}

Note: Significant differences are marked in bold.

Abbreviations: NSCLC, nonsmall-cell lung cancer; SCLC, small-cell lung cancer; WHO, World Health Organization.

had the greatest prognostic importance for survival with an HR of 1.006. ${ }^{21}$ The level of QoL should be monitored even before the diagnosis because many cancer patients experience nonspecific symptoms such as pain or couching much earlier, thus it is important to establish a prediagnosis status of QoL. Additionally, a prediagnosis level of QoL was proved to be an important predictor of survival. ${ }^{19-22}$ However, measuring a prediagnosis level of QoL in all patients suffering from nonspecific symptoms is difficult and challenging, but it would give valuable information; therefore, it essential to establish the level of QoL at least at the time of diagnosis.

Our study showed that patients with SCLC presented with lower levels of QoL and acceptance of illness than patients with NSCLC. This condition may be rooted in many factors. Primarily, SCLC patients reported worse well-being. The comparison between the groups revealed that SCLC patients had more advanced disease in terms of metastases and were more treated with combined treatment, which also reflects greater progression of metastatic disease. Disease origin, histology, treatment, and prognosis for those groups of patients add to the differences found between patients with SCLC and NSCLC. Reports from the literature show that despite the differences, there are also many similarities between those groups of patients. ${ }^{23-27}$ Therefore, we believe that comparison between SCLC and NSCLC patients can help to improve treatment strategies, especially since comparison in QoL and acceptance of illness are scarce in the literature.

QoL and symptom burden is important for lung cancer patients. Due to the severity of the disease, in most cases, treatment is aggressive, associated with treatment-induced toxicity, and often conducted with the aim to palliate symptoms, which affects patients with SCLC to a greater degree. Also, current research into lung cancer therapy is largely focused on molecular therapies for NSCLC which may improve 
Table 2 Comparison of QoL between patients with NSCLC and SCLC

\begin{tabular}{|c|c|c|c|c|c|}
\hline Variables & NSCLC & SCLC & $p$-value & $\begin{array}{l}\text { Reference } \\
\text { values NSCLC }\end{array}$ & $\begin{array}{l}\text { Reference } \\
\text { values SCLC }\end{array}$ \\
\hline \multicolumn{6}{|l|}{ QLQ-C30 functioning area } \\
\hline Global & $47.12 \pm 23.21$ & $38.3 I \pm 20.25$ & 0.009 & $58.8 \pm 22.5$ & $67.1 \pm 20.4$ \\
\hline Physical & $72.32 \pm 17.96$ & $61.02 \pm 21.13$ & $<0.001$ & $78.4 \pm 19.3$ & $78.7 \pm 18.7$ \\
\hline Role & $65.95 \pm 27.4 I$ & $51.62 \pm 28.96$ & $<0.00 \mathrm{I}$ & $60.7 \pm 33.1$ & $73.9 \pm 29.8$ \\
\hline Emotional & $57.97 \pm 28.76$ & $52.43 \pm 25.46$ & 0.171 & $68.1 \pm 24.2$ & $75.9 \pm 21.7$ \\
\hline Cognitive & $76.13 \pm 21.12$ & $68.06 \pm 24.35$ & 0.018 & $84.0 \pm 21.1$ & $85.7 \pm 19.7$ \\
\hline Social & $65.77 \pm 32.4$ & $59.49 \pm 23.21$ & 0.038 & $73.6 \pm 28.9$ & $76.0 \pm 26.1$ \\
\hline \multicolumn{6}{|l|}{ QLQ-C30 symptom area } \\
\hline Fatigue & $42.64 \pm 26.5$ I & $51.23 \pm 25.4$ & 0.016 & $40.4 \pm 27$ & $36.4 \pm 25.3$ \\
\hline Nausea/vomiting & $\mid 2.61 \pm 18.23$ & $30.32 \pm 26.88$ & $<0.001$ & $9.7 \pm 18.3$ & $9.9 \pm 17.6$ \\
\hline Pain & $36.94 \pm 23.8$ & $44.44 \pm 27.69$ & 0.033 & $29.7 \pm 30.3$ & $18.4 \pm 24.8$ \\
\hline Dyspnea & $41.62 \pm 24.4$ & $48.61 \pm 27.37$ & 0.057 & $38.5 \pm 31.7$ & $31.8 \pm 29.8$ \\
\hline Sleep disturbance & $41.98 \pm 31.43$ & $50.46 \pm 29.6$ & 0.054 & $32.4 \pm 32.7$ & $23.1 \pm 27.8$ \\
\hline Appetite loss & $31.17 \pm 28.36$ & $43.52 \pm 31.47$ & 0.004 & $27.9 \pm 33.5$ & $24.3 \pm 30.4$ \\
\hline Constipation & $17.48 \pm 26.25$ & $27.78 \pm 28.53$ & 0.002 & $17.4 \pm 27.9$ & $15.6 \pm 26.1$ \\
\hline Diarrhea & $9.19 \pm 22.65$ & $|1.57 \pm| 9.5 \mid$ & 0.065 & $6.8 \pm 17.4$ & $7.3 \pm 16$ \\
\hline Financial problems & $33.33 \pm 31.85$ & $53.7 \pm 28.83$ & $<0.001$ & $12.8 \pm 25.8$ & $17.0 \pm 28.8$ \\
\hline \multicolumn{6}{|l|}{ QLQ-LCI3 } \\
\hline Dyspnea & $34.17 \pm 22.83$ & $47.99 \pm 23.58$ & $<0.001$ & & \\
\hline Coughing & $50.09 \pm 24.11$ & $54.63 \pm 27.01$ & 0.256 & & \\
\hline Hemoptysis & $14.59 \pm 20.78$ & $19.91 \pm 24.17$ & 0.105 & & \\
\hline Sore mouth and tongue & $7.57 \pm 15.63$ & $23.15 \pm 27.2$ & $<0.001$ & & \\
\hline Dysphagia & $12.79 \pm 19.93$ & $25.93 \pm 29.19$ & $<0.001$ & & \\
\hline Peripheral neuropathy & $11.89 \pm 18.79$ & $29.63 \pm 28.28$ & $<0.001$ & & \\
\hline Hair loss & $12.07 \pm 23.92$ & $30.56 \pm 37.42$ & $<0.001$ & & \\
\hline Chest pain & $23.24 \pm 25.19$ & $41.2 \pm 29.86$ & $<0.001$ & & \\
\hline Pain in arm or shoulder & $12.79 \pm 22.49$ & $21.76 \pm 28.61$ & 0.009 & & \\
\hline Other pain sites & $23.78 \pm 27.56$ & $29.17 \pm 34.46$ & $0.45 I$ & & \\
\hline Taking analgesics & $50.4 I \pm 37$ & $49.31 \pm 31.67$ & 0.477 & & \\
\hline
\end{tabular}

Notes: Significant differences are marked in bold. according to the EORTC QLQ-C30 reference values. ${ }^{16}$

Abbreviations: NSCLC, nonsmall-cell lung cancer; SCLC, small-cell lung cancer; QoL, quality of life; QLQ-C30, European Organization for Research and Treatment of Cancer 30-item Core Quality of Life Questionnaire; QLQ-LCI3, European Organization for Research and Treatment of Cancer I3-item Lung Cancer specific Quality of Life Questionnaire.

future outcomes, but only in this group of patients. Studies revealed deficits in QoL dimensions with no improvement after introduced treatment and shorted survival in patients with SCLC. ${ }^{28}$ Our study also confirmed that QoL is worse in SCLC than in NSCLC. However, interventions with the aim to alleviate symptoms of the disease have significant impact of QoL. Pain management and cessation of smoking are associated with reduction in perceived pain, dyspnea, and fatigue. ${ }^{29}$

Studies assessing acceptance of illness in lung cancer patients are scarce. Religioni et a ${ }^{30}$ reported that patients with lung cancer had significantly lower level of acceptance of illness than patients with breast, colorectal, and prostate carcinoma. The mean AIS score for lung cancer patients was 23.17. They also found a correlation between income and AIS score, which was significant only in lung cancer patients. ${ }^{30}$ Patients from our study had slightly higher level of acceptance (27.05 for NSCLC and 24.58 for SCLC), but interestingly, in the group with SCLC, financial problems scale score was over threefold worse than reference values. Such discrepancy is alarming. It draws attention to the fact that patients with advanced lung cancer may live in poverty, which in turn decreases their QoL and chances for optimal treatment.

Our study draws attention to the fact that intensity of symptoms and QoL may vary among patients with the same diagnosis; therefore, there is a need for collection of patient-reported outcomes data with standardized tools and tailored approach to treatment. In clinical practice, patient's perspective differs from physician's assessment, which can lead to underestimating symptom severity or health condition. Efficace et $\mathrm{al}^{31}$ showed that agreement on symptom ratings between patients with chronic myeloid leukemia and their treating physicians ranges from 34\% for muscle cramps to $66 \%$ for nausea. All symptoms were more severe from patient's perspective than from physician's perspective. ${ }^{31}$ Also, attitudes to treatment options and outcomes expectations may not be the same. As reported 
Table 3 Correlations between acceptance of illness and dimensions of QoL in patients with NSCLC and SCLC

\begin{tabular}{|c|c|c|c|c|}
\hline \multirow[t]{2}{*}{ Variables } & \multicolumn{2}{|c|}{ AIS score in NSCLC patients } & \multicolumn{2}{|c|}{ AIS score in SCLC patients } \\
\hline & Correlation coefficient & p-value & Correlation coefficient & p-value \\
\hline \multicolumn{5}{|l|}{ QLQ-C30 functioning area } \\
\hline Global & 0.754 & $<0.00$ I & 0.577 & $<0.001$ \\
\hline Physical & 0.495 & $<0.00$ I & 0.62 & $<0.001$ \\
\hline Role & 0.629 & $<0.001$ & 0.666 & $<0.001$ \\
\hline Emotional & 0.742 & $<0.001$ & 0.593 & $<0.001$ \\
\hline Cognitive & 0.595 & $<0.001$ & 0.537 & $<0.001$ \\
\hline Social & 0.723 & $<0.00$ I & 0.642 & $<0.001$ \\
\hline \multicolumn{5}{|l|}{ QLQ-C30 symptom area } \\
\hline Fatigue & 0.723 & $<0.001$ & -0.658 & $<0.001$ \\
\hline Nausea/vomiting & -0.648 & $<0.001$ & -0.229 & 0.053 \\
\hline Pain & -0.396 & $<0.00$ I & -0.617 & $<0.001$ \\
\hline Dyspnea & -0.648 & $<0.001$ & -0.525 & $<0.001$ \\
\hline Sleep disturbance & -0.324 & $<0.001$ & -0.453 & $<0.001$ \\
\hline Appetite loss & -0.47 & $<0.001$ & -0.366 & 0.002 \\
\hline Constipation & -0.443 & $<0.001$ & -0.09 & 0.453 \\
\hline Diarrhea & -0.154 & 0.037 & -0.069 & 0.565 \\
\hline Financial problems & -0.187 & 0.011 & -0.182 & 0.125 \\
\hline \multicolumn{5}{|l|}{ QLQ-LCI3 } \\
\hline Dyspnea & -0.598 & $<0.001$ & -0.554 & $<0.001$ \\
\hline Coughing & -0.409 & $<0.001$ & -0.336 & 0.004 \\
\hline Hemoptysis & 0.158 & 0.032 & -0.173 & 0.147 \\
\hline Sore mouth and tongue & -0.205 & 0.005 & -0.357 & 0.002 \\
\hline Dysphagia & -0.394 & $<0.001$ & -0.048 & 0.687 \\
\hline Peripheral neuropathy & -0.328 & $<0.00$ I & -0.206 & 0.083 \\
\hline Hair loss & -0.022 & 0.765 & -0.125 & 0.294 \\
\hline Chest pain & -0.339 & $<0.00$ I & -0.254 & 0.031 \\
\hline Pain in arm or shoulder & -0.251 & 0.001 & -0.225 & 0.057 \\
\hline Other pain sites & -0.441 & $<0.001$ & -0.339 & 0.004 \\
\hline Taking analgesics & -0.049 & 0.509 & -0.123 & 0.302 \\
\hline
\end{tabular}

Note: Bold text represents a statistically significant value of $p<0.05$.

Abbreviations: AIS, Acceptance of Illness Scale; NSCLC, nonsmall-cell lung cancer; SCLC, small-cell lung cancer; QoL, quality of life; QLQ-C30, Organization for Research and Treatment of Cancer Quality of Life Questionnaire Core 30; QLQ-LCI3, Organization for Research and Treatment of Cancer Quality of Life Questionnaire Core I3.

by Chu et al, ${ }^{32}$ physicians were more inclined to set smaller goals such as relief of symptoms, whereas patients with advanced NSCLC more likely opted for extended survival with acceptance of high toxicity due to chemotherapy. These examples emphasize the role of proper dialogue between patients and their treating physicians to enable taking into consideration patients reported outcomes and using tools for their assessment broadly in clinical practice.

\section{Conclusion}

Patients with SCLC reported lower level of QoL, lower acceptance of illness, and higher intensity of pain than patients with NSCLC. Monitoring of QoL, personalized approach to treatment, goal of treatment based on mutual expectations, and interventions for symptom management may improve prognosis in this group of patients. Socioeconomic status of lung cancer patients, especially those suffering from SCLC, needs to be addressed.

\section{Acknowledgment}

This study was performed within the project of Wroclaw Medical University number ST.E020.17.050.

\section{Disclosure}

The authors report no conflicts of interest in this work.

\section{References}

1. Kreyberg L, Liebow AA, Uehlinger EA. Histological Typing of Lung Tumours. 1st ed. Geneva: WHO; 1967.

2. Travis WD, Brambilla E, Nicholson AG, et al. The 2015 World Health Organization classification of lung tumors: impact of genetic, clinical and radiologic advances since the 2004 classification. J Thorac Oncol. 2015;10(9):1243-1260.

3. Govindan R, Page N, Morgensztern D, et al. Changing epidemiology of small-cell lung cancer in the United States over the last 30 years: analysis of the surveillance, epidemiologic, and end results database. J Clin Oncol. 2006;24(28):4539-4544.

4. Sculier JP. Nonsmall cell lung cancer. Eur Respir Rev. 2013;22(127):33-36.

5. Khakwani A, Rich AL, Tata LJ, et al. Small-cell lung cancer in England: trends in survival and chemotherapy using the National Lung Cancer Audit. PLoS One. 2014;9(2):e89426. 
6. Wang S, Tang J, Sun T, et al. Survival changes in patients with small cell lung cancer and disparities between different sexes, socioeconomic statuses and ages. Sci Rep. 2017;7(1):1339.

7. Khakwani A, Rich AL, Powell HA, et al. Lung cancer survival in England: trends in non-small-cell lung cancer survival over the duration of the National Lung Cancer Audit. Br J Cancer. 2013;109(8):2058-2065.

8. Visbal AL, Williams BA, Nichols FC III, et al. Gender differences in non-small-cell lung cancer survival: an analysis of 4,618 patients diagnosed between 1997 and 2002. Ann Thorac Surg. 2004;78(1):209-215.

9. Juczyński Z. [Measurement tools in the promotion and psychoobcology of health]. Warszawa, Poland: Pracownia Testów Psychologicznych. 2009. Polish.

10. Felton BJ, Revenson TA, Hinrichsen GA. Stress and coping in the explanation of psychological adjustment among chronically ill adults. Soc Sci Med. 1984;18(10):889-898.

11. Hawker GA, Mian S, Kendzerska T, French M. Measures of adult pain: Visual Analog Scale for Pain (VAS Pain), Numeric Rating Scale for Pain (NRS Pain), McGill Pain Questionnaire (MPQ), Short-Form McGill Pain Questionnaire (SF-MPQ), Chronic Pain Grade Scale (CPGS), Short Form-36 Bodily Pain Scale (SF-36 BPS), and Measure of Intermittent and Constant Osteoarthritis Pain (ICOAP). Arthritis Care Res (Hoboken). 2011;63(Suppl 11):S240-S252.

12. Katz J, Melzack R. Measurement of pain. Surg Clin North Am. 1999;79(2):231-252.

13. Aaronson NK, Ahmedzai S, Bergman B, et al. The European Organization for Research and Treatment of Cancer QLQ-C30: a quality-of-life instrument for use in international clinical trials in oncology. $\mathrm{J} \mathrm{Natl}$ Cancer Inst. 1993;85(5):365-376.

14. Tomaszewski KA, Puskulluoglu M, Biesiada K, Bochenek J, Nieckula J, Krzemieniecki K. Validation of the polish version of the EORTC QLQ-C30 and the QLQ-OG25 for the assessment of health-related quality of life in patients with esophagi-gastric cancer. J Psychosoc Oncol. 2013;31(2):191-203.

15. Bergman B, Aaronson NK, Ahmedzai S, Kaasa S, Sullivan M. The EORTC QLQ-LC13: a modular supplement to the EORTC Core Quality of Life Questionnaire (QLQ-C30) for use in lung cancer clinical trials. Eur J Cancer. 1994;30A(5):635-642.

16. Scott NW, Fayers PM, Aaronson NK, et al. EORTC QLQ-C30 Reference Values. Brussels: EORTC Quality of Life Group; 2008. Available from: http://groups.eortc.be/qol/sites/default/files/img/newsletter/reference_ values_manual2008.pdf. Accessed September 9, 2017.

17. Tomaszewski KA, Püsküllüoğlu M, Biesiada K, Bochenek J, Nieckula J, Krzemieniecki K. Validation of the polish version of the eortc QLQC30 and the QLQ-OG25 for the assessment of health-related quality of life in patients with esophagi-gastric cancer. J Psychosoc Oncol. 2013;31(2):191-203.

18. U.S. Department of Health and Human Services FDA Center for Drug Evaluation and Research, U.S. Department of Health and Human Services FDA Center for Biologics Evaluation and Research, U.S. Department of Health and Human Services FDA Center for Devices and Radiological Health. Guidance for industry: patient-reported outcome measures: use in medical product development to support labeling claims: draft guidance. Health Qual Life Outcomes. 2006;4:79.
19. Montazeri A, Milroy R, Hole D, McEwen J, Gillis CR. Quality of life in lung cancer patients: as an important prognostic factor. Lung Cancer. 2001;31(2-3):233-240.

20. Efficace F, Bottomley A, Smit EF, et al. Is a patient's self-reported health-related quality of life a prognostic factor for survival in nonsmall-cell lung cancer patients? A multivariate analysis of prognostic factors of EORTC study 08975. Ann Oncol. 2006;17(11): 1698-1704.

21. Herndon JE 2nd, Fleishman S, Kornblith AB, Kosty M, Green MR, Holland J. Is quality of life predictive of the survival of patients with advanced nonsmall cell lung carcinoma? Cancer. 1999;85(2): 333-340.

22. Pinheiro LC, Zagar TM, Reeve BB. The prognostic value of prediagnosis health-related quality of life on survival: a prospective cohort study of older Americans with lung cancer. Qual Life Res. 2017;26(7): 1703-1712.

23. Nakazawa K, Kurishima K, Tamura T, Ishikawa H, Satoh H, Hizawa N. Survival difference in NSCLC and SCLC patients with diabetes mellitus according to the first-line therapy. Med Oncol. 2013;30(1) 367.

24. Toh CK, Gao F, Lim WT, et al. Differences between small-cell lung cancer and non-small-cell lung cancer among tobacco smokers. Lung Cancer. 2007;56(2):161-166.

25. Oser MG, Niederst MJ, Sequist LV, Engelman JA. Transformation from non-small-cell lung cancer to small-cell lung cancer: molecular drivers and cells of origin. Lancet Oncol. 2015;16(4):e165-e172.

26. Staal-van den Brekel AJ, Schols AM, Dentener MA, ten Velde GP, Buurman WA, Wouters EF. Metabolism in patients with small cell lung carcinoma compared with patients with non-small cell lung carcinoma and healthy controls. Thorax. 1997;52(4):338-341.

27. Miret M, Horváth-Puhó E, Déruaz-Luyet A, Sørensen HT, Ehrenstein V. Potential paraneoplastic syndromes and selected autoimmune conditions in patients with non-small cell lung cancer and small cell lung cancer: a population-based cohort study. PLoS One. 2017;12(8) e0181564.

28. Lithoxopoulou H, Zarogoulidis K, Bostantzopoulou S, et al. Monitoring changes in quality of life in patients with lung cancer by using specialised questionnaires: implications for clinical practice. Support Care Cancer. 2014;22(8):2177-2183.

29. Chen J, Qi Y, Wampfler JA, et al. Effect of cigarette smoking on quality of life in small cell lung cancer patients. Eur J Cancer. 2012;48(11): 1593-1601.

30. Religioni U, Czerw A, Deptala A. Acceptance of cancer in patients diagnosed with lung, breast, colorectal and prostate carcinoma. Iran J Public Health. 2015;44(8):1135-1142.

31. Efficace F, Rosti G, Aaronson N, et al. Patient- versus physicianreporting of symptoms and health status in chronic myeloid leukemia. Haematologica. 2014;99(4):788-793.

32. Chu DT, Kim SW, Kuo HP, et al. Patient attitudes towards chemotherapy as assessed by patient versus physician: a prospective observational study in advanced non-small cell lung cancer. Lung Cancer. 2007;56(3):433-443.
Journal of Pain Research

\section{Publish your work in this journal}

The Journal of Pain Research is an international, peer reviewed, open access, online journal that welcomes laboratory and clinical findings in the fields of pain research and the prevention and management of pain. Original research, reviews, symposium reports, hypothesis formation and commentaries are all considered for publication.
The manuscript management system is completely online and includes a very quick and fair peer-review system, which is all easy to use. Visit http://www.dovepress.com/testimonials.php to read real quotes from published authors. 